\title{
Caracterização agronômica e carpométrica de cultivares de oliveira ${ }^{1}$
}

\author{
Luiz Fernando de Oliveira da Silva ${ }^{2}$, Adelson Francisco de Oliveira ${ }^{3}$, Rafael $\mathrm{Pio}^{2}$, Carolina Ruiz Zambon ${ }^{4}$
}

\begin{abstract}
Agronomic and carpometric

characterization of olive tree cultivars

Growing olive trees in Brazil is a recent practice, and their productive attributes, as well as available cultivars, are not yet well-known. The agronomic and carpometric characterization of fruits from different cultivars are vital for the improvement of the Brazilian olive growing. This study aimed at evaluating the agronomic characterization and carpometry of 35 olive tree cultivars. The experiment was conducted in the Empresa de Pesquisa Agropecuária de Minas Gerais (Epamig) experimental farm, in Maria da Fé, Minas Gerais State, Brazil. For the agronomic characterization, a completely randomized design with three replications was used, with one plant per plot, and yield, number of fruits per plant, trunk section and outer crown volume and surface were evaluated. For the carpometric characterization, a completely randomized design with ten replications was used, with one fruit or pit per plot, and length, width, fruits mass and volume and pit, as well as the pulp/pit ratio, were evaluated. The agronomic features of plants differed among cultivars, standing out the Negroa, which reached the highest yield (18.42 kg plant ${ }^{-1}$ and $\left.7.68 \mathrm{t} \mathrm{ha}^{-1}\right)$ and number of fruits (9,399.08); MGS ASC322, with the largest trunk section; MGS MISS293, with the highest outer crown volume and surface; and MGS JB1, which presented the greatest outer crown surface/number of fruits ratio. Concerning carpometry, the MGS GRAP556 cultivar presented the highest sizes for fruit and pit, while 'MGS GRAP541' presented the highest pulp/pit ratio.
\end{abstract}

KEY-WORDS: Olea europaea L.; plant vigor; fruit size.

\section{INTRODUÇÃO}

O cultivo de oliveira (Olea europaea L.) é uma das principais atividades econômicas da região Mediterrânea. A Espanha produz cerca de 33\% de toda a azeitona e azeite do mundo, sendo a região de

\section{RESUMO}

O cultivo de oliveira, no Brasil, é recente e os seus atributos produtivos, bem como cultivares disponíveis, ainda não são muito conhecidos. A caracterização agronômica e carpométrica dos frutos de diferentes cultivares são primordiais para o avanço da olivicultura brasileira. Este trabalho objetivou realizar a caracterização agronômica e carpométrica de 35 cultivares de oliveira. O experimento foi conduzido na fazenda experimental da Empresa de Pesquisa Agropecuária de Minas Gerais (Epamig), em Maria da Fé (MG). Para a caracterização agronômica, foi utilizado o delineamento inteiramente casualizado, com três repetições, sendo uma planta por parcela, e a produção, produtividade, número de frutos por planta, seção de tronco, volume e superfície externa de copa foram avaliados. Para a caracterização carpométrica, foi utilizado o delineamento inteiramente casualizado, com 10 repetições, sendo um fruto ou caroço por parcela, e o comprimento, largura, massa e volume de frutos e caroços e, também, a relação polpa/caroço foram avaliados. As características agronômicas das plantas variaram entre as cultivares, com destaque para a Negroa, que apresentou maior produção $\left(18,42 \mathrm{~kg}\right.$ planta $\left.^{-1}\right)$, produtividade $\left(7,68 \mathrm{t} \mathrm{ha}^{-1}\right) \mathrm{e}$ número de frutos $(9.399,08)$; MGS ASC322, com maior seção de tronco; MGS MISS293, com maior volume e superfície externa de copa; e MGS JB1, que apresentou maior relação superfície externa de copa/número de frutos. Quanto à carpometria, a MGS GRAP556 apresentou maiores dimensões de frutos e caroços e a MGS GRAP541 maior relação polpa/caroço.

PALAVRAS-CHAVE: Olea europaea L.; vigor de plantas; dimensão de fruto.

Andaluzia responsável por cerca de $80 \%$ da produção espanhola (Galán et al. 2005).

O Brasil configura-se, no cenário, como grande importador de azeitonas e azeite, sendo 100\% dependente de importações de países como a Espanha, Chile e Argentina. No entanto, pesquisas

1. Trabalho recebido em fev./2012 e aceito para publicação em set./2012 ( ${ }^{\circ}$ registro: PAT 17342).

2. Universidade Federal de Lavras (UFLa), Departamento de Agricultura, Lavras, MG, Brasil.

E-mails: luizfernando.agronomia@gmail.com,rafaelpio@dag.ufla.br.

3. Empresa de Pesquisa Agropecuária de Minas Gerais (Epamig), Lavras, MG, Brasil.E-mail: adelson@epamig.ufla.br.

4. Empresa de Pesquisa Agropecuária de Minas Gerais (Epamig), Maria da Fé, MG, Brasil.

E-mail: carol-rzambon@hotmail.com. 
com esta frutífera vêm sendo realizadas, em olivais já introduzidos em algumas regiões dos Estados do Rio Grande do Sul (150 ha) e Minas Gerais (300 ha), com expectativa de aumento anual em torno de $50 \%$ (Vieira Neto et al. 2010, Oliveira et al. 2010a e 2012b).

Os bancos de germoplasma, além de assegurarem o patrimônio genético das espécies, possibilitam o estudo de diversas cultivares, sob as mesmas condições de cultivo. Contrariamente ao que ocorre com outras espécies frutíferas, não existem muitos trabalhos sobre ensaios comparativos com oliveiras, no Brasil. Além disto, estas possuem bom crescimento em clima tropical e subtropical, fator importante a se considerar na expansão da olivicultura, no Brasil.

A avaliação de cultivares em diversas localidades, ao longo do tempo, é uma opção importante para estimar o diferencial de respostas genotípicas a várias condições ambientais, e, assim, estimar a interação genótipo-ambiente (Aulicino et al. 2000). Dessa forma, as cultivares podem apresentar diferencial produtivo, em relação aos plantios localizados no Mediterrâneo, ou mesmo na América do Sul.

A produção é uma característica determinada por múltiplos e diferentes caracteres genéticos, como a necessidade de clima frio, incompatibilidade polén-pistilo e tamanho do fruto, influenciados, em sua expressão, pelo ambiente e técnicas de cultivo (Del Río et al. 2005a). O vigor de uma oliveira faz referência ao seu crescimento e tamanho definitivo, e, portanto, ao espaço que ocupará em uma plantação adulta, sendo, este, o principal responsável para a determinação da densidade de plantio, permitindo que o olival receba boa iluminação e, consequentemente, resulte em máxima produção (Del Río et al. 2005b).

A carpometria dos frutos e caroços de oliveira é determinante para o processo de definição do potencial de exploração da cultivar, destacando sua finalidade de uso, seja para extração de azeite ou elaboração de conservas. As características expressas nos frutos recebem influência direta da cultivar, condições climáticas, solo, tratos culturais e estágio de maturação. Além disto, o fruto e caroço apresentam características fundamentais, no processo de identificação e diferenciação de cultivares.

O presente trabalho objetivou realizar a caracterização agronômica de plantas e carpométrica de frutos e caroços de cultivares de oliveira, no sul de Minas Gerais.

\section{MATERIAL E MÉTODOS}

A presente pesquisa foi realizada entre julho de 2010 e março de 2011, com oliveiras provenientes do Banco Ativo de Germoplasma (BAG) da fazenda experimental da Empresa de Pesquisa Agropecuária de Minas Gerias (Epamig), em Maria da Fé, sul de Minas Gerais $\left(22^{\circ} 18^{\prime} \mathrm{S}, 4^{\circ} 23^{\prime} \mathrm{W}\right.$ e altitude média de $1.276 \mathrm{~m}$ ), onde a classificação climática é do tipo Cwb, segundo Köppen (Oliveira et al. 2012a).

O BAG foi instalado em 2005, com 60 genótipos diferentes de oliveira, no espaçamento $7 \times 5$. As plantas foram conduzidas de forma livre, sendo realizadas pequenas intervenções de poda, como a limpeza de ramos mal posicionados e/ou que apresentavam algum problema fitossanitário. Dentre todos os genótipos, apenas 35 floresceram, sendo, estes, selecionados para realização deste trabalho, os quais possibilitarão a realização de futuras hibridações, visando ao melhoramento genético da oliveira, em Minas Gerais. As cultivares foram: Alto D’Ouro, Arbequina, MGS ASC315, MGS ASC322, MGS ASC323, Ascolano USA, Cerignola, MGS ZAL010, MGS VIGLIONE, MGS SEVERO, MGS GRAP084, MGS NEBLINA, Cornicabra, Empeltre, Galega, MGS GRAP541, MGS GRAP550, MGS GRAP553, MGS GRAP556, MGS GRAP561, MGS GRAP575, MGS JB1, MGS MANZ215, MGS MANZ234, MGS MARIENSE, Mission, MGS MISS293, Negroa, MGS PENA SP, Picual, MGS ROP398, MGS SAL488, Santa Catalina, MGS TAF390 e MGS TAF391.

\section{Caracterização agronômica}

O experimento foi conduzido em delineamento experimental inteiramente casualizado (DIC), sendo 35 tratamentos (cultivares) com três repetições e cada parcela constituída por uma planta. Foram avaliadas a produção $\left(\mathrm{g} \mathrm{planta}^{-1}\right)$, produtividade estimada $\left(\mathrm{t} \mathrm{ha}^{-1}\right)$, número de frutos por planta, seção de tronco $\left(\mathrm{cm}^{2}\right)$, volume de copa $\left(\mathrm{m}^{3}\right)$, superfície externa de copa $\left(\mathrm{m}^{2}\right)$ e a relação número de frutos/superfície externa de copa.

Os frutos foram colhidos de cada cultivar e pesados em balança analítica, quantificando-se, assim, a produção por planta. Com estes resultados, estimou-se a produtividade, levando-se em consideração um pomar com espaçamento de $6,0 \mathrm{~m}$ x 4,0 m (417 plantas $\left.\mathrm{ha}^{-1}\right)$. 
O diâmetro de tronco foi medido com o auxílio de um paquímetro digital, a $10,0 \mathrm{~cm}$ do solo. A partir deste valor, calculou-se o perímetro do tronco e, posteriormente, sua seção, por meio da expressão $\mathrm{ST}=\pi(\mathrm{P} / 2 \pi)^{2}$, para plantas com diâmetro superior a $5,0 \mathrm{~cm}$ ou 4 anos.

Para o cálculo do volume e superfície externa de copa, foi medida a altura da copa $(\mathrm{H})$, com o auxílio de uma régua graduada, verticalmente ao lado da árvore, a partir da inserção dos primeiros ramos laterais, até o ápice do ramo mais alto, e, depois, em duas direções horizontais perpendiculares, correspondentes à sua maior (D1) e menor (D2) largura, utilizando-se as expressões $\mathrm{VC}=\pi\left((\mathrm{D} 1+\mathrm{D} 2)^{2} / 2\right) \mathrm{H} / 6$ e $\mathrm{SC}=\pi((\mathrm{D} 1+\mathrm{D} 2) / 2) \mathrm{H}$. Após este procedimento, calculou-se a relação número de frutos/superfície externa de copa.

\section{Carpometria}

O delineamento experimental utilizado foi inteiramente casualizado (DIC), com 35 cultivares e 10 repetições, com um fruto ou caroço por parcela, escolhido de forma aleatória.

Foram avaliados o comprimento ( $\mathrm{mm})$, largura $(\mathrm{mm})$, massa (g) e volume $\left(\mathrm{mm}^{3}\right)$ dos frutos e caroços. Após as avaliações, foi determinada a relação polpa/ caroço, por meio da expressão polpa $/$ caroço $=($ massa do fruto - massa do caroço)/massa do caroço.

As avaliações dos frutos e caroços foram realizadas com o auxílio de paquímetro digital (largura e comprimento), balança analítica de precisão (massa) e proveta graduada (volume), sendo medido o volume de água deslocado.

Os resultados foram submetidos à análise de variância e as médias comparadas pelo teste Scott-Knott, a 5\%, por meio do software estatístico Sisvar ${ }^{\circledR}$ (Ferreira 2011).

\section{RESULTADOS E DISCUSSÃO}

A cultivar Negroa apresentou superioridade, em relação às demais, na produção de frutos $\left(18,42 \mathrm{~kg}\right.$ planta $\left.{ }^{-1}\right)$, produtividade $\left(7,68 \mathrm{t} \mathrm{ha}^{-1}\right) \mathrm{e}$ número médio de frutos $\left(9.399,08\right.$ frutos planta $\left.^{-1}\right)$ (Tabela 1). Outras cultivares, como a Alto D'Ouro, MGS ASC315 e MGS JB1, apresentaram bom desempenho, com produção por planta acima de $10,00 \mathrm{~kg}\left(11,24 \mathrm{~kg}\right.$ planta $^{-1}, 11,57 \mathrm{~kg}$ planta $^{-1} \mathrm{e}$ $12,35 \mathrm{~kg} \mathrm{planta}^{-1}$, respectivamente).
Neste trabalho, a produção média, aos 6 anos após o plantio, variou de $0,18 \mathrm{~kg}$ planta $^{-1}$ (Empeltre) a 18,42 kg planta-1 (Negroa) (Tabela 1), resultados distintos dos encontrados por Del Río et al. (2005a), ao caracterizar a produção média do Banco de Germoplasma de Córdoba, na Espanha, onde foi registrada produção média de 2,00 kg planta ${ }^{-1}$ a 52,00 kg planta $^{-1}$, em plantas com idade média de 4,4 anos.

Esse fato sugere que as plantas, por estarem em condições climáticas distintas das mediterrâneas, podem apresentar atraso produtivo, quando comparadas às cultivadas em regiões tradicionais, sendo que a precocidade produtiva varia de acordo com a cultivar, influenciada pelo ambiente. Del Río \& Caballero (2004) observaram que a precocidade produtiva é uma característica desejada, que permite cortar a fase improdutiva e amortizar os gastos com o olival. Estes mesmos autores destacaram que existem notáveis diferenças varietais, na precocidade de produção.

A cultivar MGS ASC322 apresentou maior seção média de tronco $\left(396,94 \mathrm{~cm}^{2}\right)$, enquanto Ascolano USA $\left(58,11 \mathrm{~cm}^{2}\right)$, Cerignola $\left(67,39 \mathrm{~cm}^{2}\right)$, MGS ZAL010 $\left(66,46 \mathrm{~cm}^{2}\right)$, MGS VIGLIONE $\left(61,07 \mathrm{~cm}^{2}\right)$, MGS SEVERO $\left(58,84 \mathrm{~cm}^{2}\right)$, MGS MARIENSE $\left(65,68 \mathrm{~cm}^{2}\right)$ e MGS PENA SP $\left(56,21 \mathrm{~cm}^{2}\right)$ apresentaram as menores médias (Tabela 1).

Em relação ao volume médio e superfície externa média de copa, MGS MISS293 apresentou as maiores médias $\left(59,02 \mathrm{~m}^{3}\right.$ e $71,33 \mathrm{~m}^{2}$, respectivamente), enquanto MGS MARIENSE apresentou as menores médias $\left(6,45 \mathrm{~m}^{3}\right.$ e 19,64 $\mathrm{m}^{2}$, respectivamente), para as mesmas variáveis.

Diferentemente do que se observa com outras espécies frutíferas, como o pessegueiro e a macieira (Kreuz et al. 1996, Rocha et al. 2007), as variáveis produtividade e produção não apresentaram correlação positiva, em relação à superfície externa e volume de copa das cultivares. Apesar de uma oliveira apresentar boas condições físicas para uma boa florada, a taxa efetiva de frutificação é dependente de outros fatores, como polinização (autopolinização e fecundação cruzada), viabilidade polínica, condição climática, sanidade e estado nutricional da planta (Erel et al. 2008).

Em anos de baixa carga, o crescimento de ramos é constante, sempre que as condições forem favoráveis, não existindo limitação de água e nutrientes, e sempre que a temperatura média estiver entre $10^{\circ} \mathrm{C}$ e $30^{\circ} \mathrm{C}$, aproximadamente. Em anos de altas produções, a demanda por fotoassimilados é alta, sendo a produção de ramos menor (Rallo \& Suárez 1989). 
Tabela 1. Produção média (PDM), produtividade média estimada (PVME), número de frutos por planta (FP), seção média de tronco (SMT), volume médio de copa (VMC), superfície externa média de copa (SEMC) e relação FP/SEMC de cultivares de oliveira implantadas em Maria da Fé (MG), safra 2010/2011 (Lavras, MG, 2011).

\begin{tabular}{|c|c|c|c|c|c|c|c|}
\hline \multirow{2}{*}{ Cultivar } & PDM & PVME & \multirow{2}{*}{ NFP } & SMT & VMC & SEMC & \multirow{2}{*}{$\mathrm{NFP} / \mathrm{SMC}$} \\
\hline & kg planta $^{-1}$ & $\mathrm{t} \mathrm{ha}^{-1}$ & & $\mathrm{~cm}^{2}$ & $\mathrm{~m}^{3}$ & $\mathrm{~m}^{2}$ & \\
\hline Alto D'Ouro & $11,24 \mathrm{~b}$ & $4,69 \mathrm{~b}$ & $5.110,18 \mathrm{c}$ & $135,10 \mathrm{e}$ & $28,67 \mathrm{e}$ & $47,18 \mathrm{~d}$ & $108,46 \mathrm{c}$ \\
\hline Arbequina & $0,71 \mathrm{e}$ & $0,30 \mathrm{~d}$ & $658,98 \mathrm{e}$ & $111,81 \mathrm{f}$ & $16,38 \mathrm{~m}$ & $29,99 \mathrm{i}$ & $21,97 \mathrm{e}$ \\
\hline MGS ASC315 & $11,57 \mathrm{~b}$ & $4,83 \mathrm{~b}$ & $2.787,15 \mathrm{~d}$ & $128,02 \mathrm{e}$ & $27,21 \mathrm{f}$ & $40,67 \mathrm{e}$ & $68,73 \mathrm{~d}$ \\
\hline MGS ASC322 & $1,07 \mathrm{e}$ & $0,45 \mathrm{~d}$ & $329,29 \mathrm{e}$ & $396,94 \mathrm{a}$ & $27,33 \mathrm{f}$ & $36,96 \mathrm{f}$ & $8,90 \mathrm{e}$ \\
\hline MGS ASC323 & $1,31 \mathrm{e}$ & $0,55 \mathrm{~d}$ & $360,88 \mathrm{e}$ & $103,08 \mathrm{f}$ & 15,13 o & $32,80 \mathrm{~h}$ & $11,00 \mathrm{e}$ \\
\hline Ascolano USA & $0,92 \mathrm{e}$ & $0,39 \mathrm{~d}$ & $178,57 \mathrm{e}$ & $58,11 \mathrm{~h}$ & $7,87 \mathrm{u}$ & $22,54 \mathrm{k}$ & $7,98 \mathrm{e}$ \\
\hline Cerignola & $1,33 \mathrm{e}$ & $0,55 \mathrm{~d}$ & $176,66 \mathrm{e}$ & $67,39 \mathrm{~h}$ & $10,65 \mathrm{r}$ & $29,34 \mathrm{i}$ & $5,90 \mathrm{e}$ \\
\hline MGS ZAL010 & $1,98 \mathrm{e}$ & $0,82 \mathrm{~d}$ & $1.122,29 \mathrm{e}$ & $66,46 \mathrm{~h}$ & $27,25 \mathrm{f}$ & $49,71 \mathrm{~d}$ & $22,57 \mathrm{e}$ \\
\hline MGS VIGLIONE & $0,47 \mathrm{e}$ & $0,20 \mathrm{~d}$ & $407,36 \mathrm{e}$ & $61,07 \mathrm{~h}$ & $8,69 \mathrm{t}$ & $23,69 \mathrm{k}$ & $17,23 \mathrm{e}$ \\
\hline MGS SEVERO & $1,28 \mathrm{e}$ & $0,53 \mathrm{~d}$ & 436,14 e & $58,84 \mathrm{~h}$ & $11,57 \mathrm{q}$ & $25,35 \mathrm{j}$ & $17,20 \mathrm{e}$ \\
\hline MGS GRAP084 & $5,40 \mathrm{~d}$ & $2,25 \mathrm{c}$ & $1.666,67 \mathrm{~d}$ & $82,86 \mathrm{~g}$ & $13,84 \mathrm{p}$ & $29,64 \mathrm{i}$ & $56,25 \mathrm{~d}$ \\
\hline MGS NEBLINA & $6,20 \mathrm{~d}$ & $2,59 \mathrm{c}$ & $1.807,58 \mathrm{~d}$ & $137,52 \mathrm{e}$ & 17,141 & $30,89 \mathrm{i}$ & $58,53 \mathrm{~d}$ \\
\hline Cornicabra & $1,33 \mathrm{e}$ & $0,55 \mathrm{~d}$ & $831,25 \mathrm{e}$ & $79,81 \mathrm{~g}$ & $8,65 \mathrm{t}$ & $25,17 \mathrm{j}$ & $33,00 \mathrm{e}$ \\
\hline Empeltre & $0,18 \mathrm{e}$ & $0,07 \mathrm{~d}$ & $58,25 \mathrm{e}$ & $153,56 \mathrm{~d}$ & $15,87 \mathrm{n}$ & $34,39 \mathrm{~g}$ & $1,69 \mathrm{e}$ \\
\hline Galega & $6,54 \mathrm{~d}$ & $2,72 \mathrm{c}$ & $2.701,10 \mathrm{~d}$ & $154,72 \mathrm{~d}$ & $30,66 \mathrm{~d}$ & $47,06 \mathrm{~d}$ & $56,87 \mathrm{~d}$ \\
\hline MGS GRAP541 & $1,82 \mathrm{e}$ & $0,76 \mathrm{~d}$ & $642,11 \mathrm{e}$ & $123,73 \mathrm{e}$ & 15,20 o & $31,12 \mathrm{i}$ & $20,57 \mathrm{e}$ \\
\hline MGS GRAP550 & $0,71 \mathrm{e}$ & $0,30 \mathrm{~d}$ & $148,93 \mathrm{e}$ & $180,78 \mathrm{c}$ & $24,08 \mathrm{i}$ & $47,62 \mathrm{~d}$ & $3,12 \mathrm{e}$ \\
\hline MGS GRAP553 & $0,93 \mathrm{e}$ & $0,53 \mathrm{~d}$ & $201,66 \mathrm{e}$ & $73,30 \mathrm{~g}$ & 7,91 u & $22,92 \mathrm{k}$ & $8,80 \mathrm{e}$ \\
\hline MGS GRAP556 & $5,80 \mathrm{~d}$ & $2,42 \mathrm{c}$ & $528,16 \mathrm{e}$ & $158,79 \mathrm{~d}$ & $13,61 \mathrm{p}$ & $29,65 \mathrm{i}$ & $17,84 \mathrm{e}$ \\
\hline MGS GRAP561 & $1,40 \mathrm{e}$ & $0,58 \mathrm{~d}$ & $355,33 \mathrm{e}$ & $108,17 \mathrm{f}$ & 17,221 & $42,43 \mathrm{e}$ & $8,31 \mathrm{e}$ \\
\hline MGS GRAP575 & $0,88 \mathrm{e}$ & $0,37 \mathrm{~d}$ & $569,89 \mathrm{e}$ & $74,17 \mathrm{~g}$ & $15,68 \mathrm{n}$ & $32,81 \mathrm{~h}$ & $17,36 \mathrm{e}$ \\
\hline MGS JB1 & $12,35 \mathrm{~b}$ & $5,15 \mathrm{~b}$ & $6.604,66 \mathrm{~b}$ & $138,38 \mathrm{e}$ & $19,77 \mathrm{k}$ & $37,00 \mathrm{f}$ & $178,54 \mathrm{a}$ \\
\hline MGS MANZ215 & $1,15 \mathrm{e}$ & $0,48 \mathrm{~d}$ & $244,25 \mathrm{e}$ & $71,69 \mathrm{~g}$ & 14,96 o & $31,33 \mathrm{i}$ & $7,82 \mathrm{e}$ \\
\hline MGS MANZ234 & $0,72 \mathrm{e}$ & $0,30 \mathrm{~d}$ & $244,80 \mathrm{e}$ & $159,82 \mathrm{~d}$ & $41,75 \mathrm{c}$ & $56,14 \mathrm{c}$ & $4,37 \mathrm{e}$ \\
\hline MGS MARIENSE & $0,39 \mathrm{e}$ & $0,16 \mathrm{~d}$ & $405,19 \mathrm{e}$ & $65,68 \mathrm{~h}$ & $6,45 \mathrm{v}$ & 19,641 & $20,33 \mathrm{e}$ \\
\hline Mission & $8,03 \mathrm{c}$ & $3,35 \mathrm{c}$ & $4.160,62 \mathrm{c}$ & $171,92 \mathrm{c}$ & $26,10 \mathrm{~g}$ & $48,90 \mathrm{~d}$ & $85,74 \mathrm{c}$ \\
\hline MGS MISS293 & $1,45 \mathrm{e}$ & $0,60 \mathrm{~d}$ & $354,52 \mathrm{e}$ & $216,89 \mathrm{~b}$ & $59,02 \mathrm{a}$ & $71,33 \mathrm{a}$ & $4,90 \mathrm{e}$ \\
\hline Negroa & $18,42 \mathrm{a}$ & $7,68 \mathrm{a}$ & $9.399,08 \mathrm{a}$ & $176,97 \mathrm{c}$ & $49,53 \mathrm{~b}$ & $67,10 \mathrm{~b}$ & $139,86 \mathrm{~b}$ \\
\hline MGS PENA SP & $0,20 \mathrm{e}$ & $0,08 \mathrm{~d}$ & $113,98 \mathrm{e}$ & $56,21 \mathrm{~h}$ & $9,30 \mathrm{~s}$ & $22,49 \mathrm{k}$ & $5,12 \mathrm{e}$ \\
\hline Picual & $4,31 \mathrm{~d}$ & $1,80 \mathrm{c}$ & $886,21 \mathrm{e}$ & $124,52 \mathrm{e}$ & $25,31 \mathrm{~h}$ & $42,90 \mathrm{e}$ & $20,68 \mathrm{e}$ \\
\hline MGS ROP398 & $1,51 \mathrm{e}$ & $0,63 \mathrm{~d}$ & $273,08 \mathrm{e}$ & $218,70 \mathrm{~b}$ & $23,90 \mathrm{i}$ & $38,88 \mathrm{f}$ & $7,05 \mathrm{e}$ \\
\hline MGS SAL488 & $3,45 \mathrm{~d}$ & $1,44 \mathrm{c}$ & $2.105,69 \mathrm{~d}$ & $147,94 \mathrm{~d}$ & $13,85 \mathrm{p}$ & $30,21 \mathrm{i}$ & $69,45 \mathrm{~d}$ \\
\hline Santa Catalina & $1,34 \mathrm{e}$ & $0,56 \mathrm{~d}$ & $172,13 \mathrm{e}$ & $110,26 \mathrm{f}$ & $15,33 \mathrm{o}$ & $34,37 \mathrm{~g}$ & $5,02 \mathrm{e}$ \\
\hline MGS TAF390 & $0,90 \mathrm{e}$ & $0,38 \mathrm{~d}$ & $201,64 \mathrm{e}$ & $101,26 \mathrm{f}$ & $13,30 \mathrm{p}$ & $26,47 \mathrm{j}$ & $7,60 \mathrm{e}$ \\
\hline MGS TAF391 & $4,00 \mathrm{~d}$ & $1,67 \mathrm{c}$ & $553,25 \mathrm{e}$ & $129,69 \mathrm{e}$ & $23,14 \mathrm{j}$ & $41,59 \mathrm{e}$ & $13,31 \mathrm{e}$ \\
\hline CV $(\%)$ & 20,87 & 50,21 & 23,30 & 8,47 & 1,46 & 3,85 & 55,42 \\
\hline
\end{tabular}

Médias seguidas da mesma letra, nas colunas, não diferem entre si pelo teste Scott-Knott, a 5\%.

A cultivar MGS JB1 apresentou o melhor resultado para a relação número de frutos/superfície externa de copa $(178,54)$ (Tabela 1). A densidade de plantio de uma cultivar é determinante para assegurar uma boa iluminação e máxima produção. Além disto, o crescimento depende, fundamentalmente, da quantidade de fotoassimilados produzidos pela superfície foliar e da produção de raízes e ramos.

Quanto à carpometria dos frutos e caroços, a cultivar MGS GRAP556 apresentou as maiores dimensões para todas as características analisadas no fruto $\left(29,24 \mathrm{~mm} ; 25,00 \mathrm{~mm} ; 10,99 \mathrm{~g} ; \mathrm{e} 10,79 \mathrm{~mm}^{3}\right)$ e caroço $\left(20,43 \mathrm{~mm} ; 11,69 \mathrm{~mm} ; 1,64 \mathrm{~g}\right.$; e $\left.1,41 \mathrm{~mm}^{3}\right)$, para comprimento, largura, massa e volume, respectivamente (Tabela 2).

A cultivar MGS MARIENSE, por sua vez, apresentou as menores dimensões de fruto e caroço (respectivamente 13,44 mm e 9,14 mm de comprimento; $10,19 \mathrm{~mm}$ e $5,77 \mathrm{~mm}$ de largura; $0,95 \mathrm{~g}$ e $0,26 \mathrm{~g}$ de massa; e $0,72 \mathrm{~mm}^{3}$ e $0,29 \mathrm{~mm}^{3}$ de volume) (Tabela 3). A maior relação polpa/caroço foi encontrada na cultivar MGS GRAP541 $(13,09)$ e 
Tabela 2. Comprimento, largura, massa média, volume e relação polpa/caroço de frutos de cultivares de oliveira implantadas em Maria da Fé (MG), safra 2010/2011 (Lavras, MG, 2011).

\begin{tabular}{|c|c|c|c|c|c|}
\hline \multirow{2}{*}{ Cultivar } & Comprimento & Largura & $\begin{array}{l}\text { Massa } \\
\text { média }\end{array}$ & Volume & \multirow{2}{*}{ Polpa/caroçc } \\
\hline & \multicolumn{2}{|c|}{$\mathrm{mm}$} & $\mathrm{g}$ & $\mathrm{mm}^{3}$ & \\
\hline Alto D'Ouro & $19,29 \mathrm{~g}$ & $14,36 \mathrm{~m}$ & $2,20 \mathrm{q}$ & $2,12 \mathrm{~s}$ & $4,98 \mathrm{~h}$ \\
\hline Arbequina & $15,27 \mathrm{i}$ & $11,54 \mathrm{o}$ & $1,08 \mathrm{u}$ & $1,49 \mathrm{v}$ & 2,781 \\
\hline MGS ASC315 & $24,53 \mathrm{c}$ & $20,80 \mathrm{~d}$ & $4,15 \mathrm{j}$ & $4,31 \mathrm{j}$ & $3,54 \mathrm{j}$ \\
\hline MGS ASC322 & $23,54 \mathrm{c}$ & 15,771 & $3,24 n$ & 3,30 o & $3,69 \mathrm{j}$ \\
\hline MGS ASC323 & $21,28 \mathrm{e}$ & $17,48 \mathrm{j}$ & 3,631 & $3,55 \mathrm{~m}$ & $4,92 \mathrm{~g}$ \\
\hline Ascolano USA & $21,62 \mathrm{e}$ & $18,92 \mathrm{~h}$ & $5,17 \mathrm{f}$ & $5,36 \mathrm{~g}$ & $4,76 \mathrm{~g}$ \\
\hline Cerignola & $25,58 \mathrm{~b}$ & $22,84 \mathrm{~b}$ & $7,51 \mathrm{c}$ & $7,56 \mathrm{e}$ & $7,76 \mathrm{~b}$ \\
\hline MGS ZALM010 & $17,26 \mathrm{~h}$ & $12,80 \mathrm{n}$ & $1,76 \mathrm{~s}$ & $1,31 \mathrm{w}$ & $3,74 \mathrm{j}$ \\
\hline MGS VIGLIONE & $15,23 \mathrm{i}$ & 11,98 o & $1,16 \mathrm{u}$ & $1,19 x$ & $3,02 \mathrm{k}$ \\
\hline MGS SEVERO & $26,08 \mathrm{~b}$ & $21,49 \mathrm{c}$ & 2,93 o & $3,10 \mathrm{p}$ & $4,05 \mathrm{i}$ \\
\hline MGS GRAP084 & $20,16 \mathrm{f}$ & $16,69 \mathrm{k}$ & $3,24 \mathrm{n}$ & $3,39 \mathrm{n}$ & $4,51 \mathrm{~h}$ \\
\hline MGS NEBLINA & $22,70 \mathrm{~d}$ & $16,60 \mathrm{k}$ & $3,43 \mathrm{~m}$ & $3,41 \mathrm{n}$ & $3,72 \mathrm{j}$ \\
\hline Cornicabra & $16,70 \mathrm{~h}$ & $12,73 \mathrm{n}$ & $1,60 \mathrm{t}$ & $1,13 \mathrm{x}$ & $3,77 \mathrm{j}$ \\
\hline Empeltre & $19,85 \mathrm{f}$ & 15,261 & $3,14 \mathrm{n}$ & $2,88 \mathrm{q}$ & $6,21 \mathrm{~d}$ \\
\hline Galega & $18,38 \mathrm{~g}$ & $14,17 \mathrm{~m}$ & $2,42 \mathrm{p}$ & $2,16 \mathrm{~s}$ & $5,37 \mathrm{f}$ \\
\hline MGS GRAP541 & $18,39 \mathrm{~g}$ & 15,921 & $2,84 \mathrm{o}$ & $2,70 \mathrm{r}$ & $13,09 \mathrm{a}$ \\
\hline MGS GRAP550 & $21,17 \mathrm{e}$ & $19,92 \mathrm{e}$ & $4,79 \mathrm{~g}$ & $4,94 \mathrm{~h}$ & $6,73 \mathrm{c}$ \\
\hline MGS GRAP553 & $24,44 \mathrm{c}$ & $18,39 \mathrm{i}$ & $4,63 \mathrm{~h}$ & $4,89 \mathrm{~h}$ & $7,81 \mathrm{~b}$ \\
\hline MGS GRAP556 & $29,94 \mathrm{a}$ & $25,00 \mathrm{a}$ & $10,99 \mathrm{a}$ & $10,79 \mathrm{a}$ & $5,68 \mathrm{e}$ \\
\hline MGS GRAP561 & $21,61 \mathrm{e}$ & $18,04 \mathrm{j}$ & $3,94 \mathrm{k}$ & $4,21 \mathrm{k}$ & $5,51 \mathrm{f}$ \\
\hline MGS GRAP575 & $16,27 \mathrm{~h}$ & $12,22 \mathrm{o}$ & $1,55 \mathrm{t}$ & $1,20 \mathrm{x}$ & $3,11 \mathrm{k}$ \\
\hline MGS JB1 & $18,29 \mathrm{~g}$ & $13,86 \mathrm{~m}$ & $1,87 \mathrm{r}$ & $2,16 \mathrm{~s}$ & $3,59 \mathrm{j}$ \\
\hline MGS MANZ215 & $20,66 \mathrm{f}$ & $20,57 \mathrm{~d}$ & $4,71 \mathrm{~g}$ & 4,071 & $6,74 \mathrm{c}$ \\
\hline MGS MANZ234 & $18,54 \mathrm{~g}$ & $16,31 \mathrm{k}$ & $2,94 \mathrm{o}$ & 4,161 & $3,83 \mathrm{j}$ \\
\hline MGS MARIENSE & $13,44 \mathrm{j}$ & $10,19 \mathrm{p}$ & $0,95 \mathrm{v}$ & $0,72 \mathrm{y}$ & $2,74 \mathrm{k}$ \\
\hline Mission & $19,27 \mathrm{~g}$ & $13,89 \mathrm{~m}$ & $1,93 \mathrm{r}$ & $2,13 \mathrm{~s}$ & $3,81 \mathrm{j}$ \\
\hline MGS MISS293 & $21,36 \mathrm{e}$ & $19,01 \mathrm{~g}$ & $4,09 \mathrm{j}$ & $4,51 \mathrm{i}$ & $5,38 \mathrm{f}$ \\
\hline Negroa & $18,78 \mathrm{~g}$ & $13,62 \mathrm{~m}$ & $1,96 \mathrm{r}$ & $1,95 \mathrm{t}$ & $3,67 \mathrm{j}$ \\
\hline MGS PENA SP & $17,15 \mathrm{~h}$ & $12,69 n$ & $1,73 \mathrm{~s}$ & $1,86 \mathrm{t}$ & $3,10 \mathrm{k}$ \\
\hline Picual & $24,81 \mathrm{c}$ & $19,42 \mathrm{f}$ & $4,86 \mathrm{~g}$ & $4,89 \mathrm{~h}$ & $5,82 \mathrm{e}$ \\
\hline MGS ROP398 & $22,92 \mathrm{~d}$ & $20,52 \mathrm{~d}$ & $5,23 \mathrm{e}$ & $5,55 \mathrm{f}$ & $5,30 \mathrm{f}$ \\
\hline MGS SAL488 & $16,09 \mathrm{~h}$ & $13,56 \mathrm{~m}$ & $1,64 \mathrm{t}$ & $1,67 \mathrm{u}$ & $5,55 \mathrm{f}$ \\
\hline Santa Catalina & $26,54 \mathrm{~b}$ & $23,04 \mathrm{~b}$ & $7,76 \mathrm{~b}$ & $8,67 \mathrm{c}$ & $7,78 \mathrm{~b}$ \\
\hline MGS TAF390 & $20,65 \mathrm{f}$ & $19,39 \mathrm{f}$ & $4,47 \mathrm{i}$ & $8,96 \mathrm{~b}$ & $6,87 \mathrm{c}$ \\
\hline MGS TAF391 & $25,86 \mathrm{~b}$ & $22,53 \mathrm{~b}$ & $7,23 \mathrm{~d}$ & $7,85 \mathrm{~d}$ & $7,77 \mathrm{~b}$ \\
\hline $\mathrm{CV}(\%)$ & 7,29 & 5,99 & 3,61 & 2,68 & 5,36 \\
\hline
\end{tabular}

Médias seguidas da mesma letra, nas colunas, não diferem entre si pelo teste Scott-Knott, a 5\%.

a menor relação para a cultivar Arbequina $(2,78)$ (Tabela 2).

Oliveira et al. (2010b), ao trabalharem com carpometria de algumas cultivares de oliveira mantidas na fazenda experimental da Epamig, em Maria da Fé (MG), obtiveram resultados diferentes dos encontrados no presente trabalho, com comprimento de frutos variando de $15,01 \mathrm{~mm}$ (Arbequina) a $18,67 \mathrm{~mm}$ (Galega) e largura variando de $8,31 \mathrm{~mm}$ (Negroa) a 13,67 mm (MGS GRAP561). Já para a massa de frutos, estes autores encontraram médias variarando de 1,47 g (Arbequina) a 3,14 g (MGS GRAP541). Del Río et al. (2005a), em trabalho realizado com diversas cultivares, encontraram resultados semelhantes, para a relação polpa/caroço $(4,00$ a 13,70$)$.

As dimensões do fruto podem variar em função da cultivar, com comprimento entre $10,00 \mathrm{~mm}$ e $40,00 \mathrm{~mm}$ e largura entre $6,00 \mathrm{~mm}$ e $20,00 \mathrm{~mm}$. Ressalta-se que a média geral, para as dimensões das cultivares estudadas neste trabalho, apresentou-se dentro do intervalo encontrado nos países produtores 
Tabela 3. Comprimento, largura, massa média e volume de caroços de cultivares de oliveira implantadas em Maria da Fé (MG), safra 2010/2011 (Lavras, MG, 2011).

\begin{tabular}{|c|c|c|c|c|}
\hline \multirow{2}{*}{ Cultivar } & Comprimento & Largura & \multirow{2}{*}{$\frac{\text { Massa média }}{\mathrm{g}}$} & \multirow{2}{*}{$\frac{\text { Volume }}{\mathrm{mm}^{3}}$} \\
\hline & & 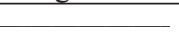 & & \\
\hline Alto D'Ouro & $14,34 \mathrm{~d}$ & $6,66 \mathrm{~h}$ & $0,39 \mathrm{n}$ & $0,38 \mathrm{i}$ \\
\hline Arbequina & $11,83 \mathrm{e}$ & $6,40 \mathrm{~h}$ & $0,29 \mathrm{p}$ & $0,38 \mathrm{i}$ \\
\hline MGS ASC315 & $15,78 \mathrm{c}$ & $9,85 \mathrm{~b}$ & $0,92 \mathrm{~b}$ & $0,63 \mathrm{e}$ \\
\hline MGS ASC322 & $16,98 \mathrm{~b}$ & $8,58 \mathrm{~d}$ & $0,69 \mathrm{~g}$ & $0,57 \mathrm{f}$ \\
\hline MGS ASC323 & $14,03 \mathrm{~d}$ & $8,61 \mathrm{~d}$ & $0,61 \mathrm{i}$ & $0,62 \mathrm{e}$ \\
\hline Ascolano USA & $17,04 \mathrm{~b}$ & $9,09 \mathrm{c}$ & $0,90 \mathrm{~b}$ & $0,77 \mathrm{c}$ \\
\hline Cerignola & $16,30 \mathrm{~b}$ & $9,13 \mathrm{c}$ & $0,86 \mathrm{~d}$ & $0,88 \mathrm{~b}$ \\
\hline MGS ZALM010 & $13,24 \mathrm{~d}$ & $6,52 \mathrm{~h}$ & $0,37 \mathrm{n}$ & $0,38 \mathrm{i}$ \\
\hline MGS VIGLIONE & $10,88 \mathrm{f}$ & $6,31 \mathrm{~h}$ & $0,29 \mathrm{p}$ & $0,29 \mathrm{j}$ \\
\hline MGS SEVERO & $14,46 \mathrm{~d}$ & $7,92 \mathrm{e}$ & $0,38 \mathrm{j}$ & $0,37 \mathrm{i}$ \\
\hline MGS GRAP084 & $13,82 \mathrm{~d}$ & $8,25 \mathrm{e}$ & $0,59 \mathrm{i}$ & $0,62 \mathrm{e}$ \\
\hline MGS NEBLINA & $16,63 \mathrm{~b}$ & $8,31 \mathrm{e}$ & $0,73 \mathrm{f}$ & $0,58 \mathrm{f}$ \\
\hline Cornicabra & $12,29 \mathrm{e}$ & $6,30 \mathrm{~h}$ & 0,34 o & $0,29 \mathrm{j}$ \\
\hline Empeltre & $14,17 \mathrm{~d}$ & $6,53 \mathrm{~h}$ & 0,441 & $0,42 \mathrm{~h}$ \\
\hline Galega & $13,41 \mathrm{~d}$ & $6,84 \mathrm{~g}$ & $0,38 \mathrm{n}$ & $0,46 \mathrm{~h}$ \\
\hline MGS GRAP541 & $9,14 \mathrm{~g}$ & $5,77 \mathrm{i}$ & $0,20 \mathrm{~s}$ & $0,29 \mathrm{j}$ \\
\hline MGS GRAP550 & $13,73 \mathrm{~d}$ & $9,21 \mathrm{c}$ & $0,62 \mathrm{i}$ & $0,40 \mathrm{i}$ \\
\hline MGS GRAP553 & $15,55 \mathrm{c}$ & $7,54 \mathrm{f}$ & $0,53 \mathrm{k}$ & $0,62 \mathrm{e}$ \\
\hline MGS GRAP556 & $20,43 \mathrm{a}$ & $11,69 \mathrm{a}$ & $1,64 \mathrm{a}$ & $1,41 \mathrm{a}$ \\
\hline MGS GRAP561 & $13,76 \mathrm{~d}$ & $8,05 \mathrm{e}$ & $0,61 \mathrm{i}$ & $0,32 \mathrm{j}$ \\
\hline MGS GRAP575 & $15,55 \mathrm{c}$ & $6,82 \mathrm{~g}$ & $0,38 \mathrm{n}$ & $0,52 \mathrm{~g}$ \\
\hline MGS JB1 & $13,73 \mathrm{~d}$ & $6,44 \mathrm{~h}$ & $0,41 \mathrm{~m}$ & $0,50 \mathrm{~g}$ \\
\hline MGS MANZ215 & $12,12 \mathrm{e}$ & $9,10 \mathrm{c}$ & $0,61 \mathrm{i}$ & $0,75 \mathrm{c}$ \\
\hline MGS MANZ234 & $12,28 \mathrm{e}$ & $8,82 \mathrm{c}$ & $0,61 \mathrm{i}$ & $0,57 \mathrm{f}$ \\
\hline MGS MARIENSE & $9,14 \mathrm{~g}$ & $5,77 \mathrm{i}$ & $0,26 \mathrm{q}$ & $0,29 \mathrm{j}$ \\
\hline Mission & $14,31 \mathrm{~d}$ & $6,57 \mathrm{~h}$ & $0,40 \mathrm{~m}$ & $0,38 \mathrm{i}$ \\
\hline MGS MISS293 & $13,50 \mathrm{~d}$ & $8,67 \mathrm{~d}$ & $0,64 \mathrm{~h}$ & $0,48 \mathrm{~g}$ \\
\hline Negroa & $14,03 \mathrm{~d}$ & $6,78 \mathrm{~g}$ & 0,421 & $0,33 \mathrm{j}$ \\
\hline MGS PENA SP & $13,45 \mathrm{~d}$ & $7,31 \mathrm{f}$ & 0,421 & $0,61 \mathrm{e}$ \\
\hline Picual & $16,51 \mathrm{~b}$ & $9,03 \mathrm{c}$ & $0,72 \mathrm{f}$ & $0,69 \mathrm{~d}$ \\
\hline MGS ROP398 & $14,74 \mathrm{c}$ & $9,31 \mathrm{c}$ & $0,88 \mathrm{c}$ & $0,85 \mathrm{~b}$ \\
\hline MGS SAL488 & $10,66 \mathrm{f}$ & $6,25 \mathrm{~h}$ & $0,25 \mathrm{q}$ & $0,27 \mathrm{j}$ \\
\hline Santa Catalina & $16,28 \mathrm{~b}$ & $8,93 \mathrm{c}$ & $0,89 \mathrm{c}$ & $0,85 \mathrm{~b}$ \\
\hline MGS TAF390 & $13,59 \mathrm{e}$ & $8,79 \mathrm{c}$ & $0,57 \mathrm{j}$ & $0,71 \mathrm{~d}$ \\
\hline MGS TAF391 & $16,45 \mathrm{~b}$ & $8,98 \mathrm{c}$ & $0,83 \mathrm{e}$ & $0,82 \mathrm{~b}$ \\
\hline $\mathrm{CV}(\%)$ & 8,02 & 5,86 & 4,26 & 9,70 \\
\hline
\end{tabular}

europeus, corroborando os resultados obtidos por Oliveira et al. (2012b).

O conhecimento destes resultados é importante, pois, a partir deles, pode-se definir a finalidade de uso dos frutos. Para a produção de conservas (azeitonas de mesa), existe preferência para azeitonas com maior relação polpa/caroço, ou seja, maior polpa e menor caroço. Já para azeitonas destinadas à extração de azeite, há preferência por frutos com menores dimensões, pois estes apresentam maiores teores de óleo. Além disto, Del Río et al. (2005a) afirmam que a relação polpa/caroço influencia no rendimento final do azeite, já que pode existir de 3\% a $4 \%$ de azeite no caroço.
Nesse sentido, as cultivares Cerignola, MGS GRAP541, MGS GRAP553, Santa Catalina e MGS TAF391 são indicadas para a produção de conservas. $\mathrm{Na}$ indicação de cultivares para a extração de azeite, faz-se necessária uma avaliação do teor lipídico de cada uma, mesmo que estas apresentem características carpométricas favoráveis.

\section{CONCLUSÕES}

1. As características agronômicas das plantas avaliadas variaram entre as cultivares, com destaque para a cultivar Negroa, que apresentou maior produção, produtividade e número de frutos; MGS ASC322, 
com maior seção de tronco; MSG MISS293, com maior volume e superfície externa de copa; e MGS JB1, que apresentou a maior relação número de frutos/superfície externa de copa.

2. Quanto à carpometria, MGS GRAP556 apresentou as maiores dimensões (comprimento, largura, massa e volume), tanto de frutos quanto de caroços, enquanto MGS GRAP541 apresentou a maior relação polpa/caroço.

\section{AGRADECIMENTOS}

À Empresa de Pesquisa Agropecuária de Minas Gerais (Epamig), pelo apoio físico e genético, à Fundação de Amparo à Pesquisa do Estado de Minas Gerais

(Fapemig - CAG APQ 02721/09), pelo apoio financeiro na execução deste trabalho, e à Coordenação de Aperfeiçoamento de Pessoal de Nível Superior (Capes), pela concessão de bolsa de mestrado ao primeiro autor.

\section{REFERÊNCIAS}

AULICINO, M. B. et al. Análisis de la interacción genotipo-ambiente para rendimiento forrajero en cebadilla criolla. Investigación Agraria: série producción y protección vegetales, Madrid, v. 15, n. 3, p. 169-180, 2000.

DEL RÍO, C.; CABALLERO, J. Caracterización agronómica preliminar de las variedades introducidas en el banco de germoplasma de olivo de Córdoba en 1987. Fruticultura Profesional, Madrid, v. 62, n. 1, p. 9-15, 2004.

DEL RÍO, C. et al. Produccíon. In: RALLO, L. et al. (Eds.). Variedades de olivo en España. Sevilla: Mundi-Prensa, 2005a. p. 259-274.

DEL RÍO, C. et al. Vigor. In: RALLO, L. et al. (Eds.). Variedades de olivo en España. Sevilla: Mundi-Prensa, 2005b. p. 249-256.

EREL, R. et al. Flowering and fruit set of olive trees in response to nitrogen, phosphorus, and potassium. Journal of the American Society for Horticultural Science, Alexandria, v. 133, n. 5, p. 639-647, 2008.

FERREIRA, D. F. Sisvar: a computer statistical analysis system. Ciência e Agrotecnologia, Lavras, v. 35, n. 6, p. 1039-1042, 2011.

GALÁN, C. et al. Heat requirement for the onset of the Olea europaea L. pollen season in several sites in Andalusia and the effect of the expected future climate change. International Journal of Biometeorology, Amsterdam, v. 49, n. 3, p. 184-188, 2005.
KREUZ, C. L.; PETRI, J. L.; MONDARDO, M. Capacidade produtiva de macieiras cv. Fuji em diversos espaçamentos com o uso de mudas livres de viroses. Pesquisa Agropecuária Brasileira, Brasília, DF, v. 31, n. 2, p. 121-127, 1996.

OLIVEIRA, M. C. et al. Enraizamento de estacas de oliveira submetidas à aplicação de fertilizantes orgânicos e AIB. Ciência e Agrotecnologia, Lavras, v. 34, n. 2, p. 337-344, 2010a.

OLIVEIRA, A. F. et al. Espaçamento entre plantas no desempenho de jardim clonal de cultivares de oliveira. Scientia Agraria, Curitiba, v. 11, n. 4, p. 317-322, 2010 b.

OLIVEIRA, M. C. et al. Características fenológicas e físicas e perfil de ácidos graxos em oliveiras no sul de Minas Gerais. Pesquisa Agropecuária Brasileira, Brasília, DF, v. 17, n. 1, p. 30-35, 2012a.

OLIVEIRA, A. F. et al. Variação na qualidade do azeite em cultivares de oliveira. Bragantia, Campinas, v. 71, n. 2, p. 202-209, 2012b.

RALLO, L.; SUARÉZ, M. P. Seasonal dristribution of dry matter within the olive fruit-bearing limb. Advances in Horticultural Science, Fienze, v. 2, n. 1, p. 55-59, 1989.

ROCHA, M. S. et al. Comportamento agronômico inicial da cv. Chimarrita enxertada em cinco porta-enxertos de pessegueiro. Revista Brasileira de Fruticultura, Jaboticabal, v. 29, n. 3, p. 583-588, 2007.

VIEIRA NETO, J. et al. Formulações comerciais de fertilizantes foliares na finalização de mudas de variedades de oliveira. Ciência Agronômica, Fortaleza, v. 42, n. 1, p. 125-131, 2010. 